\title{
An Unusual Case of Nasal Septal Hematoma Caused by Local Dental Anesthesia in a 15-Year-Old Girl
}

\author{
Narges Alizadeh (iD ${ }^{1,}{ }^{*}$ and Sahar Paryab ${ }^{2}$ \\ ${ }^{1}$ Faculty of Medicine, Qom University of Medical Sciences, Qom, Iran \\ ${ }^{2}$ Aliabad Katoul Azad University, Aliabad Katoul, Iran \\ "Corresponding author: Faculty of Medicine, Qom University of Medical Sciences, Qom, Iran. Tel: +98-9120525635, Email: narali1360@gmail.com
}

Received 2020 October 10; Revised 2020 December 21; Accepted 2020 December 23.

\begin{abstract}
Introduction: Nasal septal hematoma (NSH) is a complication of nasal trauma, which its emergency treatment is drainage of hematoma. Delayed treatment may result in necrosis of the nasal cartilage and saddle nose deformity.

Case Presentation: Here, we reported a 15-year-old girl presented with NSH associated with a dental local anesthetic injection, which led to nasal septum necrosis.

Conclusions: Computerized tomography (CT) scanning was performed to determine the hematoma size and existence of other complications.
\end{abstract}

Keywords: Nasal Septal Hematoma, Dental Anesthesia, Hematoma, The Dental Procedure

\section{Introduction}

Nasal septal hematoma (NSH) is a rare complication of nasal trauma or surgery. The nasal septum is a thin cartilaginous layer covered by perichondrium and mucosa. Nasal trauma can damage perichondrium and submucosal vessels resulting in filling the space between cartilage and perichondrium with blood (1). Local anesthesia is one of the most important techniques of pain control in dentistry. It may cause adverse effects such as anxiety, syncope, autonomic imbalance, pain, infection, longterm sensory changes, facial nerve paralysis, hematoma, needle breakage, osteonecrosis, and eye complications (2). Hematoma may occur during injection due to venous or arterial laceration in the lower eyelid, chin area, and mouth (3).

In the present study, we described a girl with NSH who had received a dental local anesthetic injection three days before observing the symptoms of NSH. The patient was referred to the hospital with nasal pain, septal swelling, and nasal obstruction. To the best of our knowledge, the nasal septal hematoma due to local dental anesthesia has never been previously reported

\section{Case Presentation}

A 15-year-old girl presented to our hospital with a 72h history of nasal obstruction, pain, and external nasal swelling. She had no fever, nasal discharge, and headache, and no history of direct trauma to her nose or head. There was a history of a dental local anesthetic injection three days before the occurrence of NSH. She complained of severe pain at the nasolabial fold, and nasal bone appeared after dental anesthesia. Intranasal examination revealed anterior bilateral nasal septal swelling and erythema. Her paranasal sinus (PNS) CT scan showed a septal hematoma with cartilage necrosis (Figure 1). The septal hematoma was drained with a single incision. Two days later, the nasal drain was removed, and the patient was discharged with oral antibiotics. The septum was healed without perforation.

\section{Discussion}

$\mathrm{NSH}$ is the collected blood in the space between the nasal septum and perichondrium. Nasal trauma or dental surgery may cause NSH. Most of the nasal septal hematoma cases occur during infancy and childhood. The hematoma should be drained urgently unless it will cause necrosis of the nasal cartilage, saddle nose deformity, and superimposed abscess formation. NSH can cause sudden onset of nasal pain, obstruction, and nasal dorsal swelling as well as fever in the first hours (1). The physical examination, especially anterior rhinoscopy, is the main diagnostic technique of $\mathrm{NSH}$, in addition to the presence of a swollen, erythematous, and swollen nasal septum, which completely or partially blocks anterior nasal airways (4). Pain control 

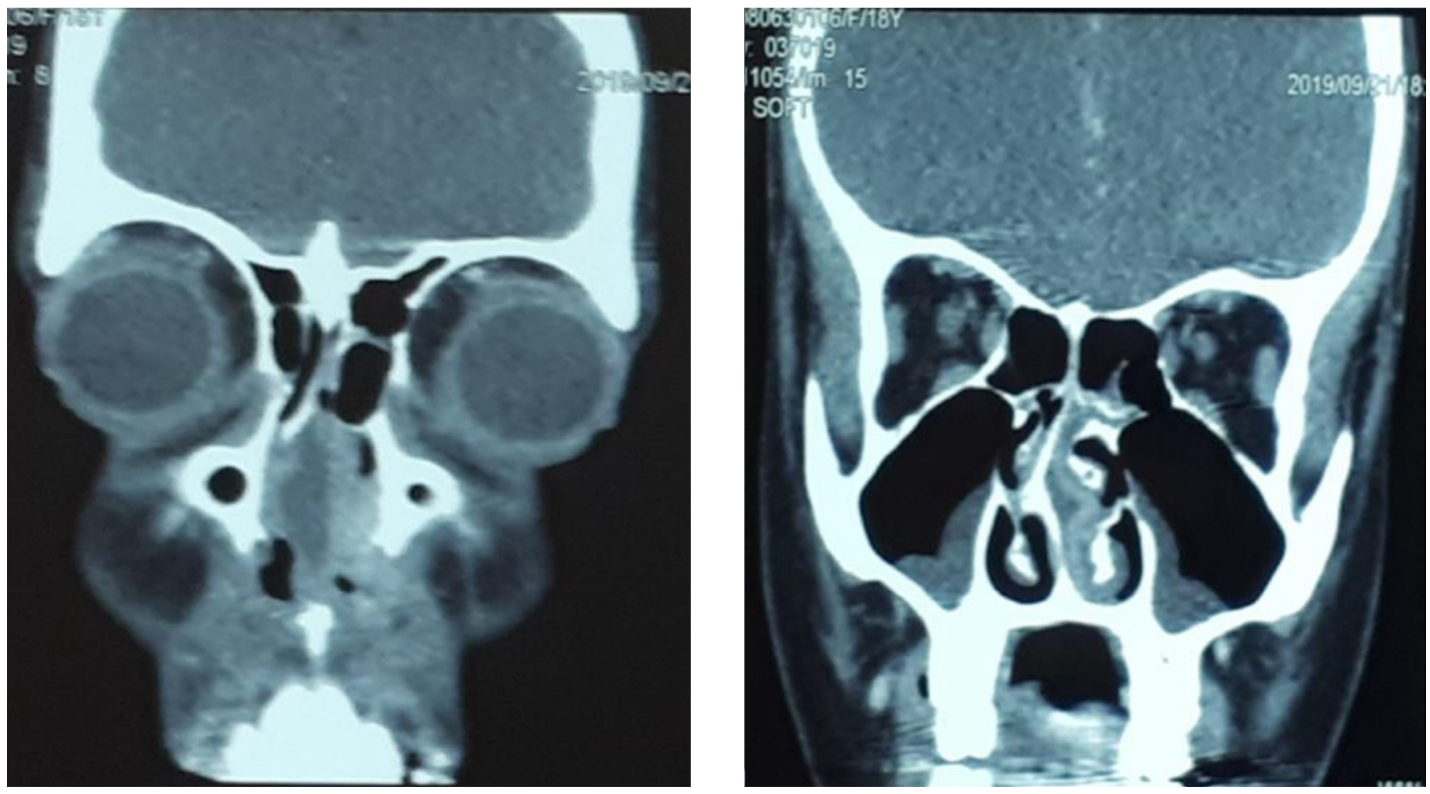

Figure 1. Coronal CT scan demonstrating septal hematoma and cartilage necrosis

during dental procedures is challenging. The most common technique for pulpal anesthesia is sub-mucosal anesthetic injection near the apex of the tooth. This procedure is painful, especially after the injection. Some supplemental anesthetic techniques, such as interosseous, intraligamentary, interdental, and intrapulpal, are available. Local anesthesia can cause complications like pain, infection, long-term sensory changes, facial nerve paralysis, trismus, orbital damage (e.g., visual loss and diplopia), osteonecrosis, hematoma, and needle breakage (5). The needle used for local anesthesia may cause venous or arterial laceration, which in turn results in the formation of hematoma in the lower eyelid, chin area, and mouth (6). However, no study has reported nasal septal hematoma due to anesthesia injection.

\subsection{Conclusion}

This is a unique case of NSH in a young child due to local dental anesthesia three days before her referral to the hospital. Many children develop NSH due to facial injuries. We should be concerned about other complications when injecting in upper teeth, such as nasal septal hematoma. Besides, it worth noting that delayed diagnosis may result in severe complications like septal cartilage necrosis (7).

\section{Footnotes}

Authors' Contribution: Study concept and design: S P, and N A; Analysis and interpretation of data: S P, and N A; Drafting of the manuscript: S P; Critical revision of the manuscript for important intellectual content: S P, NA; Statistical analysis: $\mathrm{NA}$.

Conflict of Interests: The authors declare no conflict of interest.

Funding/Support: No support.

Informed Consent: Signed by the participant.

\section{References}

1. Maharaj S, Bhaga H. An unusual collection: Nasal septal abscess secondary to a furuncle. Ear Nose Throat J. 2020:145561320961197. doi: 10.1177/0145561320961197. [PubMed: 33021836].

2. Malamed SF. Handbook of local anesthesia-e-book. Elsevier Health Sciences; 2019.

3. Biocic J, Brajdic D, Peric B, Danic P, Salaric I, Macan D. A large cheek hematoma as a complication of local anesthesia: Case report. Acta Stomatol Croat. 2018;52(2):156-9. doi: 10.15644/asc52/2/9. [PubMed: 30587858]. [PubMed Central: PMC6301842].

4. Mooney CP, Rimmer J. Spontaneous nasal septal haematoma and abscess: a case report and literature review. Rhinology Online. 2018;1(1):122-6. doi: 10.4193/rhinol/18.075.

5. Yalcin BK. Complications associated with local anesthesia in oral and maxillofacial surgery. Topics in Local Anesthetics. IntechOpen; 2019.

6. Borner U, Anschuetz L, Kaiser N, Rieke A, Dubach P, Caversaccio M. Blunt nasal trauma in children: a frequent diagnostic challenge. Eur Arch Otorhinolaryngol. 2019;276(1):85-91. doi: 10.1007/s00405-018-51831. [PubMed: 30382396].

7. Kopacheva-Barsova G, Arsova S. The Impact of the Nasal Trauma in Childhood on the Development of the Nose in Future. Open Access Maced J Med Sci. 2016;4(3):413-9. doi: 10.3889/oamjms.2016.081. [PubMed: 27703565]. [PubMed Central: PMC5042625]. 